\title{
From Classical to Contemporary Problems of Archives and Archivists
}

\author{
Peter Pavel Klasinc, Ass. Prof. Dr. \\ Archival councillor, Head: Departement of Archives and Records Management at Alma Mater Europaea, ECM, \\ Director: International Institute for Archival Science of Trieste/Maribor \\ e-mail: peter.klasinc@guest.arnes.si
}

Archivists and archives are every day exposed to new challenges. The archivists are always expected to solve classical and contemporary problems, which are arising in archival institutions and are mostly associated with the archival and current record management. Archivists are monitoring documents from their creation and at that point it is not important on what medium they are or in what way they are written. Documents are monitored by recording, by the practical use of the information contained in the documents and by the appraisal and determination of the categories of archival records. Archivists are faced with the responsibility, when they have to cope with the above and many other situations through the processes of classical or contemporary approaches.

Today we are in a situation, where the approaches of archival and record management flow into a common working process, because the functioning of the archives and the development of archival science could not be imagined otherwise, as in the symbiosis between classical and modern approaches to current problems.

Those mentioned issues are not of such nature, so the archivists would be occupied by them over their ability to manage, yet this mentioned symbiosis must be positioned with the corresponding liabilities to the processes that need to be compatible throughout the archival theory and practice.

In record management at creators, where the archival records are actually created, today we are witnessing the existence of three positions.

The first one contains classical records, which are created and stored on traditional paper-based method with records data (descriptions, indexes, registers), which may be in the classical form, and recently also in digital form.

The second position is a combination of a classical and electronic records, where is very complicated to develop a bond between both forms of records.

The third position is the so-called electronic system of record management, which is been introducing lately by many record creators. Here we meet with the term of long-term preservation.

It is the right thing to look into the past and see that tabularius, archivist in the time of the Roman Empire, and later a medieval scribe (notarius, scriptor) and today's new age archivist few decades ago did not know the term "long-term preservation " (although they were actually implementing that all the time), English words, very topical, which are today often used in archival theory and practice. Nevertheless, till the present time a large amount of classic archival records is preserved, which causes the underlying problems facing archives and archivists, and these are also reflected in the research of archival science.

In the society of the information technology archives and archivists have to deal with:

- different writing methods of electronic archival records,

- with careful record management,

- appraisal,

- conservation,

- rewriting and

- copying of electronic records,

- especially with all forms of implementation of proper material protection. 
The rapid movements in archival theory and practice, which we are also witnessing in the field of archival science can not and should not be separated from the classical traditional situations in the archives and archival science in general. Many years ago Dr. Michael Duchein drew our attention to this with the statement, that in archival theory and practice continually comes to major or minor changes, new ways of recording, archival records creation and management, but archivists should always observe in all times in changes the basic requirements of archival science, archival theory and practice, and the foundations of the existence of archival institutions.

Due to the long existence, archives themselves suffer the most when there are rapid changes in the surroundings, in which they operate, for example: the formation or abolition of independent countries or uncertainties at archival records creators, in the crises and suchlike.

It is true that archives can not affect to some fast changes, but they can professionally respond to and analyze all the consequences, which these changes are bringing along.

Archivists also have big responsibility to regulated professional tasks, determined by the law on archives and archival records or in different instructions, recommendations, manuals and such, which are prepared by the International Council on Archives, such as: Code of archival ethics, the Declaration on Archives, prescribed various standards, etc. All this imposes many obligations to archivists.

About forty years ago, we spent a lot of time in discussing on the introduction and the importance of microfilm, which was the most up to date media at the time, which has emerged as a "deus ex machina" in the hope that it will solve problems (of preservation, accessibility) related to archival records in professional archives as well as archival and current records in the archives of record creators. Microfilm was entering the professional archives through the "last" door, mainly as a way and a form of insurance against possibly destruction of archival records or in the use of archival records, when the user is offered instead of the original a microfilm copy. In many countries, the microfilm was introducing in archival theory and practice for decades and I am sure that it stayed as such until today and it is suitable for digitization.

There are many new media, which are incredibly fast "born" and even faster "dying".

Modern scanners and digitization, enabling the formation of electronic archives, are offered us as means to solve all the problems of archives and the problems relating to archival and current records. Thus, to archives incredibly huge potentials are opening, which are offered by new information technologies.

Every day we are facing the introduction of new information technologies, modern approaches to solving, maintenance or use of archival records, issues of traditional professional tasks to classical archival records and ways of preserving of large volumes of archival records, which are preserved by professional archives.

But we must not exclude the installation of systems that will technically also protect the records, which is on electronic or similar media, and which is not easy because they have actually developed from year to year, as always, new and more recent media.

By copying and transferring or by direct entering the data into a new large database, the situation became matured and it is directing us to the formation of centralized electronic archives. Many countries, which also legally defined the public electronic archival and current records, decided to establish such electronic archives.

There are useful and applicable laws and instructions in connection with the ISO 14721 from 2012 as well as the Open Archival Information System (OAIS short). As a reference model it has been implemented in some smaller countries, such as in Slovenia in the Electronic Archival Project (@ ARH.si). Within the archival system is provided preservation of digital archival records in the form of archival information packages that enable the functionality of the digital preservation of digital archival records and ensure its integrity, accessibility, usability and authenticity.

Project @ archive is implemented in many European countries, whether it this a word about a centralization, the future will show. Regional archives and archives outside the country's centre must 
have in the @ archives their indisputably independent place, it also must be enabled them to be unrestricted access, for which they also must have suitable electronic devices in their premises.

If we gave this introduction a title From classical to contemporary problems of archives and archival science, it is right to conclude the findings:

1. that in adopting decisions of any changes in relation to the bases of archival science it is necessary to reconsider these changes and to predict both positive and negative changes,

2. archivists must not fall under the dependence of IT in archives,

3. to try to avoid in archives the introduction of large information systems, and at the same time not to abandon appraisal issues,

4. according to the collected classical archival records, those records in archives should be for many years arrange it and properly maintained,

5. that the archival records created by our ancestors and was founded on the traditional carriers or new media, shall be preserved "in loco", principally, where was created;

6. that researchers need to have as easier access to the use of archival records,

7. that the symbiosis between the professional and technical solutions in the archives is imminent,

8. that it is necessary to carry out the quality professional archival work because this is the main task of archivists, wherever they operate in the world.

\section{Dai problemi tradizionali per gli archivi e gli archivisti a quelli attuali}

Archivisti ed archivi sono quotidianamente esposti a nuove sfide. Gli archivisti sono sempre ritenuti capaci di risolvere i problemi classici e contemporanei che stanno sorgendo nelle istituzioni archivistiche, per lo più associati con la gestione archivistica e corrente dei record. Gli archivisti stanno monitorando i documenti dalla loro creazione e, a quel punto non è importante su quale supporto siano o in che modo siano scritti. I documenti sono monitorati mediante registrazione, mediante l'uso pratico delle informazioni contenute nei documenti e la valutazione e la determinazione delle categorie dei documenti d'archivio. Gli archivisti fronteggiano le responsabilità, quando devono far fronte alle suddette ed a molte altre situazioni attraverso i processi di approccio classico o contemporaneo.

Oggi ci troviamo in una situazione in cui gli approcci di archiviazione e di gestione documentale confluiscono in un processo di lavoro comune, perché il funzionamento degli archivi e lo sviluppo della scienza archivistica non potevano immaginare altrimenti, come nella simbiosi tra gli approcci classici e moderni ai problemi.

I detti problemi non sono di natura tale per cui gli archivisti dovrebbero essere occupati nella loro gestione, ma la simbiosi deve prevedere dei processi compatibili con la teoria e la pratica archivistica.

Nella gestione documentale da parte dei soggetti produttori, dove i documenti d'archivio sono in realtà prodotti, si assiste oggi all'esistenza di tre posizioni.

La prima riguarda documenti tradizionali, che vengono prodotti su supporto cartaceo, con dati (descrizioni, indici, registri), che possono essere nella forma classica, e recentemente anche in quella digitale.

La seconda posizione è una combinazione di documenti tradizionali ed elettronici, dove è molto complicato sviluppare un legame tra le due forme di documenti.

La terza posizione è il cosiddetto sistema elettronico di gestione dei documenti, che è stato introdotto di recente da molti soggetti produttori e qui incontriamo il concetto di conservazione a lungo termine. 
È giusto guardare al passato e vedere che il tabularius, archivista al tempo dell'Impero romano, e poi uno scriba medievale (notarius, scriptor) e l'archivista contemporaneo fino a pochi decenni fa non conoscevano il termine "conservazione a lungo termine" (anche se sono andati effettivamente attuandolo tutto il tempo). Le parole inglesi, di grande attualità, sono oggi spesso utilizzate nella teoria e nella pratica archivistica. Tuttavia, fino ad oggi è stata conservata una grande quantità di documenti d'archivio tradizionali, il che causa i problemi di fondo che affrontano archivi ed archivisti, e questi che si riflettono anche sulla ricerca di archivio.

Nella società digitale archivi ed archivisti devono occuparsi di:

- diversi metodi di scrittura dei documenti elettronici,

- un'attenta gestione documentale,

- valutazione,

- conservazione,

- riscrittura e

- copia dei documenti elettronici,

- in particolare di tutte le idonee forme di attuazione della protezione del materiale.

La rapida evoluzione dell'archivistica teorica e pratica, come anche della scienza archivistica, non possono e non devono essere separati dalle situazioni tradizionali negli archivi e nell'archivistica in generale. Molti anni fa, il dottor Michael Duchein ha attirato la nostra attenzione su ciò, ammonendo che nella pratica e teoria archivistica si addiviene continuamente a cambiamenti principali o secondari, nuovi modi di registrazione, creazione di documentazione d'archivio e gestione, ma gli archivisti dovrebbero osservare sempre tutte le volte i requisiti fondamentali della scienza archivistica, dell'archivistica teorica e pratica e le fondamenta dell'esistenza di istituzioni archivistiche.

Gli archivi che testimoniano lunghi periodi storici, risentono dei cambiamenti istituzionali quali ad esempio la formazione o l'abolizione di paesi indipendenti o dell'incertezza dei soggetti produttori durante i periodi di transizione.

$\grave{E}$ anche vero che gli archivi non possono essere interessati a cambiamenti veloci, ma possono analizzare tutte le conseguenze, che questi cambiamenti stanno portando con sé.

Gli archivisti hanno anche grande responsabilità sui regolamenti delle attività professionali, determinate dalla legislazione sugli archivi e la documentazione d'archivio o nelle diverse istruzioni, consigli, manuali e simili che sono preparati dal Consiglio internazionale degli archivi, come: il Codice etico, la Dichiarazione sugli archivi, le varie norme, ecc. Tutto questo impone molti obblighi per gli archivisti.

Circa quarant'anni fa, abbiamo passato un sacco di tempo a discutere l'introduzione e l'importanza del microfilm, che è stata il mezzo all'epoca più aggiornato, che è emerso come un "deus ex machina" nella speranza che avrebbe potuto risolvere i problemi (di conservazione, accessibilità) legati alla archiviazione dei documenti nell'archivio professionale nonché dei documenti storici e correnti nell'archivio dei creatori. Il microfilm stava entrando negli archivi professionali attraverso la "ultima" porta, principalmente come un modo e una forma di assicurazione contro possibili distruzioni del materiale archivistico o nell' uso del materiale archivistico, quando all'utente è offerta invece dell'originale una copia in microfilm. In molti Paesi, il microfilm è stato introdotto nella teorica e pratica archivistica per decenni e sono sicuro che come tale è rimasto fino ad oggi ed è adatto per la digitalizzazione.

Ci sono molti nuovi media, che "nascono" velocemente ed ancor più velocemente "muoiono". I moderni scanner e la digitalizzazione, consentendo la formazione di archivi elettronici, ci sono offerti come mezzo per risolvere tutti i problemi degli archivi storici e contemporanei. Così, per gli archivi si stanno aprendo potenzialità incredibilmente enormi, che sono offerte dalle nuove tecnologie dell'informazione.

Ogni giorno ci troviamo di fronte all'introduzione di nuove tecnologie dell'informazione, moderni approcci per risolvere, gestire o usare il materiale archivistico, e modi di conservazione di grandi volumi di atti, che sono conservati dagli archivi professionali. 
Non dobbiamo però escludere l'installazione di sistemi che tecnicamente proteggano i documenti che siano nati su supporti elettronici o simili, il che non è facile perché in realtà si sono sviluppati anno dopo anno, come sempre nuovi e più recenti file multimediali.

Copiando e trasferendo o inserendo direttamente i dati in un nuovo database di grandi dimensioni, la situazione è evoluta fino ad indirizzarci verso la formazione di archivi elettronici centralizzati. Molti Paesi hanno anche legalmente definito la documentazione pubblica storica e corrente elettronica, decidendo di istituire archivi elettronici.

Ci sono leggi utili ed applicabili e istruzioni in relazione all'ISO 14721 del 2012, così come l'Open Archival Information System (OAIS in breve). Come modello di riferimento è stato implementato in alcuni paesi più piccoli, come ad esempio in Slovenia nel progetto di archiviazione elettronica (@ - ARH.si). All'interno del sistema archivistico si offre la conservazione del materiale archivistico digitale sotto forma di pacchetti di informazioni d'archivio che permettono la funzionalità della conservazione del materiale archivistico digitale e ne garantiscono la sua integrità, accessibilità, usabilità e autenticità.

Progetti di archivi elettronici sono implementati in molti Paesi europei, e se si dimostreranno validi per la centralizzazione lo dirà il futuro. Gli archivi regionali e quelli non statali debbono avere archivi elettronici indipendenti, inoltre deve essere permesso loro di essere ad accesso illimitato, per cui devono avere anche idonei strumenti elettronici nelle loro sedi.

Se abbiamo dato a questa introduzione il titolo "Dai problemi tradizionali per gli archivi e gli archivisti a quelli attuali", è giusto concludere che:

1. all'adozione delle decisioni di eventuali modifiche in relazione alle basi della scienza archivistica è necessario riconsiderare queste modifiche e prevederne i cambiamenti sia positivi che negativi,

2. gli archivisti non devono cadere sotto la dipendenza della tecnologia informatica negli archivi,

3. bisogna cercare di evitare l'introduzione negli archivi di sistemi informatici di grandi dimensioni ed allo stesso tempo non abbandonare i problemi legati alla valutazione,

4. come per i documenti tradizionali d'archivio, quelli informatici dovrebbero essere ordinati e conservati adeguatamente nel tempo,

5. la documentazione d'archivio prodotta nel passato e redatta su supporto tradizionale o deve essere conservata la dove è stata prodotta;

6. i ricercatori devono avere facilità di accesso all'uso del materiale archivistico,

7. la collaborazione tra le soluzioni professionali e tecniche negli archivi è imminente,

8. è necessario svolgere un lavoro di archiviazione professionale e di qualità perché questo è il compito principale degli archivisti, ovunque essi operino nel mondo.

\section{Od klasičnih do sodobnih problemov arhivov in arhivistov}

Arhivisti in arhivi so iz dneva $\mathrm{v}$ dan izpostavljeni vedno novim izzivom, od arhivistov se vedno znova pričakuje, da bodo reševali klasične in sodobne probleme, ki se vedno znova odpirajo v arhivskih ustanovah in so $\mathrm{v}$ glavnem povezani s poslovanjem $\mathrm{z}$ arhivskim in dokumentarnim gradivom. Arhivisti spremljajo dokumente od njihovega nastanka in pri tem ni pomembno na kakšni podlagi ali na kakšen način so zapisani. Dokumente spremljajo preko evidentiranja, praktične uporabe informacij, ki so zapisane v dokumentih, do njihovega vrednotenja in določanja kategorij arhivskega gradiva. Arhivisti so postavljeni pred odgovornost, ko morajo zgoraj naštete in še mnoge druge situacije obvladovati s procesi klasičnih ali sodobnih pristopov.

Danes smo v situaciji, ko se pristopi poslovanja $\mathrm{z}$ arhivskim in dokumentarnim gradivom zlivajo $\mathrm{v}$ skupen delovni proces, saj si delovanja arhivov in razvoja arhivske znanosti ne moremo predstavljati 
drugače, kot v simbiozi med klasičnimi in sodobnimi pristopi k aktualnim problemom.

Našteti problemi niso take narave, da bi okupirali arhiviste preko njihovih zmožnosti, a vendar si moramo omenjeno simbiozo zastaviti z odgovarjajočimi obveznostmi do procesov, ki morajo biti kompatibilni v celotni arhivski teoriji in praksi.

Pri vsem poslovanju z dokumenti pri ustvarjalcih, torej tam, kjer arhivsko gradivo nastaja, smo danes priče obstoja treh pozicij.

Prva vsebuje klasično gradivo, ki je nastalo in se hrani na tradicionalni papirni način z informacijami (popisi, indeksi, delovodniki) o gradivu, ki so lahko v klasični obliki, v zadnjem času pa tudi v digitalni obliki.

Druga pozicija je kombinacija klasičnega in elektronskega gradiva, pri tej je zelo zakomplicirano vzpostaviti vez med obema.

Tretja pozicija je samo tako imenovani elektronski dokumentni sistem, ki ga uvajajo v zadnjem času mnogi ustvarjalci. Tu se srečamo s terminom dolgo dobno varovanje.

Prav je, da se ozremo v preteklost in ugotovimo, da tabularius, arhivist v času Rimskega imperija in kasneje srednjeveški pisar (notarius, scriptor) ter današnji novodobni arhivist še pred nekaj desetletji niso poznali termina "dolgo dobno varovanje (čeprav so to dejansko ves čas izvajali), zelo aktualnih angleških besed, ki jih danes prepogosto uporabljamo v arhivski teoriji in praksi "long term perservation«. Kljub temu so se do današnjih dni ohranile velike količine klasičnega arhivskega gradiva, ki povzročajo osnovne probleme s katerimi se srečujejo arhivi in arhivisti ter se ti odražajo tudi v raziskavah arhivske znanosti.

V informacijski družbi se arhivi in arhivisti morajo ukvarjati;

- $\quad$ z različnimi načini zapisov elektronskega arhivskega gradiva,

- s skrbnim vodenjem,

- vrednotenjem,

- ohranjanjem,

- prepisovanjem in

- kopiranjem elektronskega gradiva,

- predvsem pa z vsemi oblikami izvajanja pravilnega materialnega varstva.

Hitrim premikom v arhivski teoriji in praksi, ki smo jim priča tudi na področju arhivistike ne moremo in ne smemo ločiti od klasičnih tradicionalnih situacij $\mathrm{v}$ arhivih in $\mathrm{v}$ arhivski znanosti na sploh. Pred mnogimi leti nas je na to opozoril dr. Michael Duchein in sicer, da v arhivski teoriji in praksi kontinuirano prihaja do večjih ali manjših sprememb, novih načinov zapisov, nastajanja in poslovanja $\mathrm{z}$ arhivskim gradivom, a vendar morajo arhivisti pri spremembah vedno upoštevati osnovne zahteve arhivske znanosti, arhivske teorije in prakse ter temelje obstoja arhivskih ustanov.

Arhivi že sami po sebi zaradi dolgega obstoja trpijo največ takrat, kadar prihaja do hitrih sprememb okolice kjer delujejo, kot so npr.: nastajanje ali ukinjanje samostojnih držav ali negotove situacije pri ustvarjalcih ob kriznih situacij in podobno.

Res je, da na nekatere hitre spremembe arhivi ne morejo vplivati, lahko pa se strokovno odzovejo in analizirajo vse posledice, ki jih takšne ali drugačne spremembe prinašajo.

Veliko odgovornost imajo arhivisti tudi do predpisanih strokovnih opravil zapisanih $\mathrm{v}$ zakonih o arhivih in arhivskem gradivu ali v raznih navodilih, priporočilih, priročnikih in podobno, kar pripravlja Mednarodni arhivski svet, kot so: Kodeks arhivske etike, Deklaracija o arhivih, predpisani razni standardi in podobno. Vse to arhivistom nalaga kar veliko obveznosti.

Pred približno štiridesetimi leti smo ogromno časa porabili za diskusije o uvajanju in pomenu mikrofilma, ki je bil takrat najbolj aktualen medij, ki se je pojavil kot »deus ex machina «v upanju, da bo rešil probleme (varovanja, dostopnosti) v zvezi z arhivskim gradivom v profesionalnih arhivih, kakor tudi arhivsko in dokumentarno gradivo pri ustvarjalcih arhivskega gradiva. V profesionalne arhive je mikrofilm prihajal skozi »zadnja« vrata, v glavnem kot način in oblika zavarovanja arhivskega gradi- 
va pred eventualnim uničenjem ali pa pri uporabi arhivskega gradiva, ko je uporabniku namesto originala ponujena mikrofilmska kopija. V mnogih državah se je mikrofilm v arhivski teoriji in praksi desetletja uveljavljal in prepričan sem, da se je kot takšen ohranil do današnjih dni in je primeren za digitalizacijo.

Pojavljajo pa se še mnogi drugi novi nosilci informacij, ki se neverjetno hitro »rojevajo« in še hitreje »umirajo«.

Sodobni skenerji in digitalizacija, ki omogočajo nastanek elektronskih arhivov se nam ponujajo kot načini s katerimi bomo rešili vse probleme arhivov in probleme $\mathrm{v}$ zvezi $\mathrm{z}$ arhivskim in dokumentarnim gradivom. Tako se arhivom odpirajo neverjetno velike možnosti, ki jih nudijo nove informacijske tehnologije.

Iz dneva $\mathrm{v}$ dan se srečujemo $\mathrm{z}$ uvajanjem novih informacijskih tehnologij, s sodobnimi pristopi do reševanja, vzdrževanja ali uporabe arhivskega gradiva, z vprašanji tradicionalnih strokovnih opravil do klasičnega arhivskega gradiva in $\mathrm{z}$ načini varovanja velikih količin arhivskega gradiva, ki ga hranijo profesionalni arhivi.

Ne smemo pa izključevati postavitve sistemov, ki bodo tehnično zavarovali tudi gradivo, ki je na elektronskih ali podobnih medijih, kar pa ni enostavno, ker so se ti razvijali pravzaprav iz leta v leto kot vedno novi in novejši nosilci informacij.

$S$ kopiranjem in prenosom ali z direktnim vnosom podatkov v nove velike baze podatkov je dozorela situacija, ki nas napotuje $\mathrm{k}$ formiranju centraliziranih elektronskih arhivov. Za te se že odločajo mnoge države, ki so zakonsko definirale javno elektronsko arhivsko in dokumentarno gradivo.

Obstoja uporabna in veljavna zakonodaja, ter navodila, ki so v zvezi z standardom ISO 14721 iz leta 2012 kakor tudi Odprt arhivski informacijski sistem (kratko OAIS). Kot referenčni model je bil uveljavljen tudi v nekaterih manjših državah kot na primer tudi v Sloveniji v Projektu elektronski arhiv (@-ARH.si). V okviru arhivskega sistema je zagotovljena hramba arhivskega digitalnega gradiva v obliki arhivskih informacijskih paketov, ki omogočajo funkcionalnost ohranjanja arhivskega digitalnega gradiva in zagotavljajo njegovo celovitost, dostopnost, uporabnost in avtentičnosti.

Projekt @ arhiv se izvaja v mnogih evropskih državah, ali gre pri tem za centralizacijo bo pokazala prihodnost. Regionalni arhivi in arhivi izven centra države morajo imeti v @ arhivu svoje nesporno samostojno mesto, omogočen pa jim mora biti neoviran dostop, za kar morajo imeti v svojih prostorih tudi primerne elektronske naprave.

Če smo temu uvodnemu razmišljanju dali naslov od klasičnih do sodobnih problemov arhivov in arhivske znanosti, je prav, da zaključimo z ugotovitvami:

1. da je pri odločitvah o kakršnih koli spremembah v zvezi z temelji arhivske znanosti potrebno o teh spremembah razmisliti in predvideti tako pozitivne kot negativne rezultate sprememb,

2. da arhivisti ne smejo pasti pod odvisnost informatikov v arhivih,

3. da se poskušamo v arhivih izogniti uvajanju velikih informacijskim sistemov in pri tem ne opustiti vprašanja vrednotenja,

4. da bo v arhivih, glede na zbrano klasično arhivsko gradivo, le tega še vrsto let potrebno urejati in primerno vzdrževati,

5. da se bo arhivsko gradivo, ki so ga ustvarili naši predniki in je nastalo na klasičnih ali novih nosilcih informacij ohranilo »in loco«, načeloma tam, kjer je tudi nastajalo,

6. da morajo imeti raziskovalci čim lažji dostop do uporabe gradiva,

7. da je simbioza med strokovnimi in tehničnimi rešitvami v arhivih neizbežna,

8. da je arhivsko strokovno delo potrebno opravljati kvalitetno, ker je to glavna naloga arhivistov, kjer koli po svetu delujejo. 